\title{
Identification and characterization of inorganic-phosphate-solubilizing bacteria from agricultural fields with a rapid isolation method
}

\author{
Bang-Xiao Zheng ${ }^{1,2,3,4+}$, Muhammad Ibrahim ${ }^{1,2+}$, Ding-Peng Zhang ${ }^{5}$, Qing-Fang Bi ${ }^{1,6}$, Hong-Zhe Li ${ }^{1,2}$, \\ Guo-Wei Zhou ${ }^{7}$, Kai Ding ${ }^{1}$, Josep Peñuelas ${ }^{3,4}$, Yong-Guan Zhu ${ }^{1,7}$ and Xiao-Ru Yang ${ }^{1 *}$
}

\begin{abstract}
The ability to solubilize fixed inorganic phosphorus (P) for plant growth is important for increasing crop yield. More $\mathrm{P}$ can be released by inoculating soil with inorganic-phosphate-solubilizing bacteria (iPSBs). We used 96-well microplates instead of traditional 200-mm petri dishes to rapidly screen iPSB strains for their solubilizing ability. We simultaneously obtained 76 iPSB isolates from 576 wells containing two agricultural soils. This method conveniently identified positive iPSB strains and effectively prevented fungal cross-contamination. Maximum-likelihood phylogenetic trees of the isolated strains showed that Bacillus megaterium was the most dominant iPSB, and strains Y99, Y95, Y924 and $\mathrm{Y} 1412$ were selected as representatives for the analysis of $\mathrm{P}$ solubilization. Succinic acid was the main organic acid of B. megaterium for releasing P. It was strongly correlated with the increase in soluble P concentration during $168 \mathrm{~h}$ of incubation of these four strains. $\mathrm{pH}$ was negatively exponentially correlated with the amount of soluble $\mathrm{P}$ in the medium, and the amount of succinic acid was strongly linearly correlated with the amount of $P$ released $(P<0.001)$, suggesting that organic acid may mobilize microbial P. Our study provides an efficient and effective method for identifying and analyzing the growth of iPSB strains able to solubilize inorganic $P$ and gives a better understanding of the mechanism of P solubilization.
\end{abstract}

Keywords: Phosphorus, Inorganic phosphate solubilizing bacteria, Isolation, Characterization

\section{Introduction}

Phosphorus (P), a non-renewable macronutrient, plays an essential role in plants (Elser et al. 2007). Inorganic $\mathrm{P}$ is mined to produce chemical $\mathrm{P}$ fertilizers that are extensively applied to cropland (Elser and Bennett 2011; Penuelas et al. 2013). The majority of soluble inorganic $\mathrm{P}$, however, is rapidly immobilized by soil fixation and becomes unavailable for plant uptake, leading to low P-use efficiency and potentially excess P (Kochian 2012).

\footnotetext{
*Correspondence: xryang@iue.ac.cn

${ }^{\dagger}$ Bang-Xiao Zheng and Muhammad Ibrahim contributed equally to this work

${ }^{1}$ Key Laboratory of Urban Environment and Health, Institute of Urban Environment, Chinese Academy of Sciences, Xiamen 361021, People's Republic of China

Full list of author information is available at the end of the article
}

Soil $\mathrm{P}$ must thus be managed to minimize its loss and increase its use efficiency.

Microorganisms are actively involved in many biogeochemical processes, including the mineralization, solubilization and transformation of soil P (van der Heijden et al. 2008). Inorganic-phosphate-solubilizing bacteria (iPSBs) are particularly effective in releasing $\mathrm{P}$ from pools of inorganic P. iPSBs can also prevent the liberated P from being fixed again (Richardson et al. 2009; Richardson and Simpson 2011). Screening highly efficient iPSBs as soil inoculum is a useful method for improving plant growth and yield (Richardson et al. 2009). Many methods for screening iPSBs have been reported (Chen et al. 2006; Chung et al. 2005; Mehta and Nautiyal 2001; Nautiyal 
1999), but isolating iPSBs on separate petri dishes can be time-consuming and labor-intensive.

The mechanism of inorganic-P microbial mobilization is generally associated with extrusion of low-molecularweight organic acids (Goldstein 1995), which can competitively chelate the cations bound to $\mathrm{P}$ via hydroxyl and carboxyl groups and convert them into soluble forms (Jones and Oburger 2011; Richardson and Simpson 2011). The solubilization of inorganic $P$, however, is complex and depends on numerous factors such as soil properties, plant nutritional requirements and physiological and growth conditions. Studies of the factors affecting solubilization are thus still needed.

We developed a rapid method for screening iPSBs and evaluating their effectiveness at solubilizing inorganic $\mathrm{P}$, with an emphasis on potential highly efficient iPSBs for agricultural use. The isolated iPSB strains were identified and characterized. The types of organic acids secreted by the iPSB strains with high P-solubilizing abilities and the relationships between the organic acids, $\mathrm{pH}$ and $\mathrm{P}$ solubilization were analyzed.

\section{Materials and methods}

\section{Soil sampling and characterization}

Soil samples were collected from agricultural fields near Hailun in Heilongjiang Province $\left(47^{\prime} 26^{\prime \prime} \mathrm{N}, 126^{\prime} 38^{\prime \prime} \mathrm{E}\right)$ and Yingtan in Jiangxi Province $\left(28^{\prime} 14^{\prime \prime} \mathrm{N} 116^{\prime} 54^{\prime \prime} \mathrm{E}\right)$, China (Table 1). Approximately $500 \mathrm{~g}$ of surface soil $(0-15 \mathrm{~cm})$ was collected after crop harvests in June 2014. The soils were then air-dried, sieved $(0.2 \mathrm{~mm})$ and stored at $4{ }^{\circ} \mathrm{C}$ until analysis.

Soil $\mathrm{pH}$ was measured using a 1:2.5 $(\mathrm{w} / \mathrm{v})$ suspension of dry soil: water and a XL60 pH meter (Fisher Scientific, USA) (Shen et al. 2008). The amounts of total $P$ and available $\mathrm{P}$ (Olsen $\mathrm{P})$ were determined using the molybdate-blue method (Murphy and Riley 1962) and sodium bicarbonate extraction (Olsen et al. 1954) after acid digestion (Parkinson and Allen 1975), respectively. The inorganic-P concentration was measured by shaking $0.2 \mathrm{~g}$ of soil in $20 \mathrm{~mL}$ of $1 \mathrm{M} \mathrm{HCl}$ at $200 \mathrm{rpm}$ for $30 \mathrm{~min}$, followed by centrifugation at $4200 \mathrm{~g}$ for $10 \mathrm{~min}$. The amount of inorganic $\mathrm{P}$ in the supernatant was then measured by the molybdate-blue method. The amount of organic P was calculated by subtracting the inorganic-P concentration from the amount of total P.

\section{Rapid screening of iPSB strains}

The iPSBs were rapidly screened using 96-well microplates (Fig. 1). A modified Pikovskaya medium (PVK) without yeast extract (Nautiyal 1999) was used as the culture medium and was added to each well in advance. The PVK was supplemented with $10 \mu \mathrm{M}$ bromocresol purple as an indicator. For each microplate, $1 \mathrm{~g}$ of soil was homogenized with $100 \mathrm{~mL}$ of sterilized water, and this suspension was then serially diluted $\left(10-10^{5}\right)$. One microliter of diluted soil suspension was added to each well and then incubated at $30{ }^{\circ} \mathrm{C}$ for at least $72 \mathrm{~h}$. An uninoculated well served as a control. Wells with no bacterial growth were considered negative. Wells with bacterial growth but no obvious color change were classified as containing uncertain strains, and wells with obvious bacterial growth and a yellow color were classified as positive. Three replicates of each dilution were tested. The effectiveness of this method was verified by streaking both uncertain and positive strains on plates containing solid modified PVK.

\section{Biochemical characterization of the iPSB strains}

The $\mathrm{pH}$ and soluble-P concentration of the medium after incubation were used as indices for iPSB screening. The indices were measured by incubating all strains in $50 \mathrm{~mL}$ of liquid modified PVK (without agar and indicator) at $30{ }^{\circ} \mathrm{C}$ for $72 \mathrm{~h}$. The supernatants obtained after centrifugation ( $4200 \mathrm{~g}$ for $10 \mathrm{~min}$ ) were used to measure $\mathrm{pH}$ with a XL60 pH meter (Fisher Scientific, USA) and phosphate concentration using the molybdate-blue method (Murphy and Riley 1962).

\section{Phylogenetic identification with $16 \mathrm{~S}$ rRNA sequencing}

All positive strains were incubated in liquid modified PVK (without indicator) at $30{ }^{\circ} \mathrm{C}$ for $24 \mathrm{~h}$. The full lengths ( $1500 \mathrm{bp})$ of $16 \mathrm{~S}$ rRNA genes were obtained by PCR using universal bacterial primers (24F: AGAGTTTGATCCTGGCTCAG and 1492R: TACGGYTACCTTGTTACGACTT) (Farris and Olson 2007). Each 50- $\mu \mathrm{L}$ PCR reaction contained $1 \mu \mathrm{L}$ of Premix Ex Taq Hot Start Version (TAKARA, Dalian, China), $0.2 \mu \mathrm{M}$ each primer and $1 \mu \mathrm{L}$ of bacterial culture suspension as DNA template. The amplification protocol was an initial denaturation at $95{ }^{\circ} \mathrm{C}$ for $4 \mathrm{~min}, 30$ cycles of $95{ }^{\circ} \mathrm{C}$ for $30 \mathrm{~s}, 58{ }^{\circ} \mathrm{C}$ for $90 \mathrm{~s}$ and $72{ }^{\circ} \mathrm{C}$ for $30 \mathrm{~s}$ and a final 5 -min extension at $72{ }^{\circ} \mathrm{C}$. The amplicons were purified with a Universal

Table 1 Basic information and soil properties of the two soil samples

\begin{tabular}{|c|c|c|c|c|c|c|}
\hline Sample & Location & $\mathrm{pH}$ & Total $\mathrm{P}\left(\mathrm{g} \mathrm{kg}^{-1}\right)$ & Olsen $\mathrm{P}\left(\mathrm{mg} \mathrm{kg}^{-1}\right)$ & Organic $\mathrm{P}\left(\mathrm{mg} \mathrm{kg}^{-1}\right)$ & Inorganic $\mathrm{P}\left(\mathrm{mg} \mathrm{kg}^{-1}\right)$ \\
\hline Hailun & $47^{\prime} 26^{\prime \prime} \mathrm{N}, 126^{\prime} 38^{\prime \prime} \mathrm{E}$ & $5.70 \pm 0.08$ & $779.35 \pm 44.33$ & $58.80 \pm 2.64$ & $351.15 \pm 6.37$ & $428.21 \pm 37.96$ \\
\hline Yingtan & $28^{\prime} 14^{\prime \prime} \mathrm{N} 116^{\prime} 54^{\prime \prime} \mathrm{E}$ & $5.01 \pm 0.03$ & $522.60 \pm 7.54$ & $23.56 \pm 1.53$ & $370.57 \pm 5.50$ & $152.04 \pm 2.05$ \\
\hline
\end{tabular}



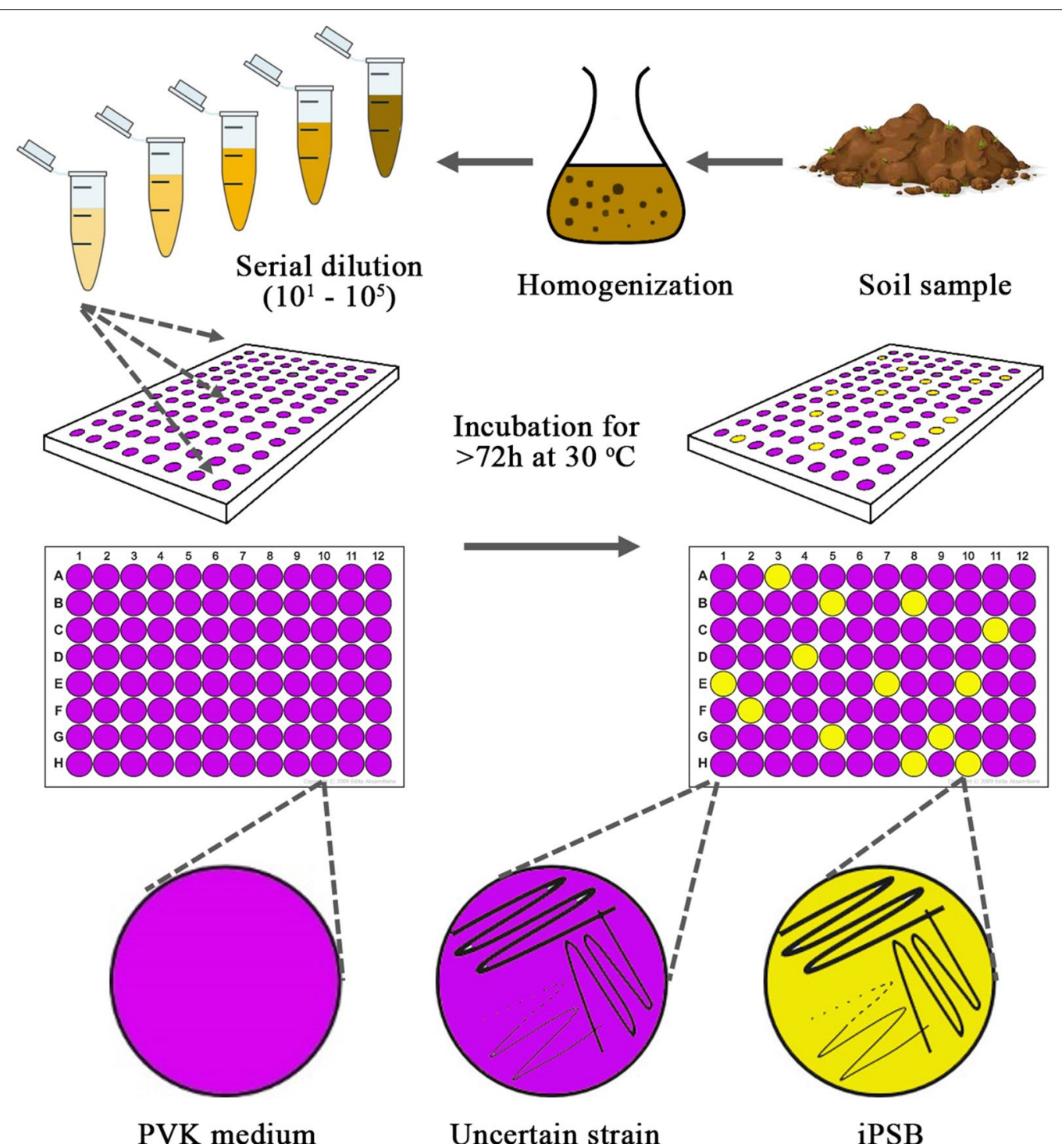

Fig. 1 Flowchart of the 96-well iPSB screening method. The sampled soil was homogenized in autoclaved water and serially diluted for incubation at $30^{\circ} \mathrm{C}$ for $>72 \mathrm{~h}$ in 96 -well microplates containing PVK with bromocresol purple as an indicator. Wells with no color change or strain growth, or with strain growth but no color change, were classified as containing unknown strains without P-solubilizing ability. Wells that produced a yellow color were classified as containing iPSB strains

DNA Purification Kit (TIANGEN, Beijing, China) and submitted for sequencing (Invitrogen, Shanghai, China). The sequences were aligned with those from bacterial lineages in GenBank at the National Center for Biotechnology Information (NCBI) (http://www.ncbi.nlm.nih.gov/) with the BLAST program. The 16S rRNA sequences of all strains were uploaded to the NCBI Sequence Read Archive with Accession Numbers KU647195-KU647270 (Table 2).

\section{Growth analysis of representative iPSB strains}

The four iPSB strains with the highest P-solubilizing capacity were used as representatives for further analysis (Bacillus megaterium Y99 was stored in China Center for Type Culture Collection, CCTCC, No. CCTCC AB 2017149). The representative strains were inoculated into $100 \mathrm{~mL}$ of liquid modified PVK and incubated at $30^{\circ} \mathrm{C}$ for $168 \mathrm{~h}$. The solubilized-P concentration and organic acid production were measured at 12,24 , 48, 96 and $168 \mathrm{~h}$. The solubilized-P concentration was determined by the molybdate-blue method as described 
Table 2 Accession numbers, medium $\mathrm{pH}$ and soluble-P concentrations of the iPSB strains after incubation for 72 h, and the closest reference strains

\begin{tabular}{|c|c|c|c|c|c|c|}
\hline \multirow[t]{2}{*}{ Strain } & \multirow[t]{2}{*}{ Accession number } & \multirow[t]{2}{*}{$\mathrm{pH}$ of medium } & \multirow{2}{*}{$\begin{array}{l}\text { Soluble-P concentra- } \\
\text { tion }\left(\mu \mathrm{g} \mathrm{mL}^{-1}\right)\end{array}$} & \multicolumn{3}{|l|}{ Closest reference strain } \\
\hline & & & & Affiliation & Accession number & Similarity (\%) \\
\hline $\begin{array}{l}\text { Bacillus megaterium } \\
01-\mathrm{A} 3\end{array}$ & KU647195 & 4.80 & 85.57 & Bacillus sp. BS3(2015) & KR063183 & 99 \\
\hline $\begin{array}{l}\text { Bacillus megaterium } \\
\text { 02-A7 }\end{array}$ & KU647196 & 4.59 & 89.08 & Bacillus sp. KU6 & JF895481 & 99 \\
\hline $\begin{array}{l}\text { Pseudomonas frederiks- } \\
\text { bergensis 03-D2 }\end{array}$ & KU647197 & 5.21 & 64.28 & Pseudomonas sp. WS06 & JN210901 & 99 \\
\hline $\begin{array}{l}\text { Rhodococcus opacus } \\
\text { 04-OD7 }\end{array}$ & KU647198 & 5.17 & 28.06 & $\begin{array}{l}\text { Rhodococcus opacus } \\
\text { DSM } 43205\end{array}$ & LN827919 & 99 \\
\hline $\begin{array}{l}\text { Arthrobacter phen- } \\
\text { anthrenivorans } \\
\text { 05-OD11 }\end{array}$ & KU647199 & 5.89 & 12.24 & $\begin{array}{l}\text { Arthrobacter phenan- } \\
\text { threnivorans L43 }\end{array}$ & LN890039 & 99 \\
\hline $\begin{array}{l}\text { Arthrobacter defluvii } \\
\text { 06-OD12 }\end{array}$ & KU647200 & 8.34 & 59.11 & $\begin{array}{l}\text { Uncultured bacterium } \\
\text { D1-57 }\end{array}$ & KC554872 & 99 \\
\hline $\begin{array}{l}\text { Arthrobacter chlorophe- } \\
\text { nolicus 07-OD13 }\end{array}$ & KU647201 & 5.58 & 20.84 & Arthrobacter sp. M29 & KF430812 & 99 \\
\hline $\begin{array}{l}\text { Arthrobacter oxydans } \\
\text { 08-OY2 }\end{array}$ & KU647202 & 6.64 & 3.85 & $\begin{array}{l}\text { Uncultured bacterium } \\
\text { D1-57 }\end{array}$ & KC554872 & 99 \\
\hline $\begin{array}{l}\text { Arthrobacter sp. } \\
\text { 09-OY5 }\end{array}$ & KU647203 & 5.11 & 43.00 & Arthrobacter sp. WSO3 & JN210899 & 99 \\
\hline $\begin{array}{l}\text { Bacillus megaterium } \\
\text { 10-Y11 }\end{array}$ & KU647204 & 4.77 & 106.46 & $\begin{array}{l}\text { Bacillus megaterium } \\
\text { HNS88 }\end{array}$ & KF933685 & 99 \\
\hline $\begin{array}{l}\text { Pseudomonas frederiks- } \\
\text { bergensis 11-D3 }\end{array}$ & KU647205 & 5.25 & 81.76 & $\begin{array}{l}\text { Pseudomonas sp. } \\
\text { B3039 }\end{array}$ & KC236870 & 99 \\
\hline Massilia putida 12-OD1 & KU647206 & 4.63 & 97.29 & $\begin{array}{l}\text { Uncultured bacterium } \\
\text { clone HF31 }\end{array}$ & KR188907 & 99 \\
\hline Duganella sp. 13-D4 & KU647207 & 5.69 & 10.78 & Duganella sp. ZLP-XI & KF896136 & 99 \\
\hline $\begin{array}{l}\text { Bacillus megaterium } \\
14-Y 2\end{array}$ & KU647208 & 4.75 & 101.58 & $\begin{array}{l}\text { Bacillus megaterium } \\
\text { Y20 }\end{array}$ & JQ798391 & 99 \\
\hline $\begin{array}{l}\text { Pseudoduganella sp. } \\
15-Y 6\end{array}$ & KU647209 & 5.29 & 49.64 & $\begin{array}{l}\text { Pseudoduganella sp. } \\
\text { NI28 }\end{array}$ & KM087999 & 99 \\
\hline $\begin{array}{l}\text { Bacillus megaterium } \\
\text { 16-Y9 }\end{array}$ & KU647210 & 4.66 & 80.20 & Bacillus sp. RBB1 & GU979225 & 99 \\
\hline $\begin{array}{l}\text { Bacillus megaterium } \\
17-Y 5\end{array}$ & KU647211 & 4.85 & 80.39 & $\begin{array}{l}\text { Uncultured Bacillus sp. } \\
\text { clone T7F50d237 }\end{array}$ & JN187411 & 99 \\
\hline $\begin{array}{l}\text { Variovorax paradoxus } \\
\text { 19-D4 }\end{array}$ & KU647212 & 5.42 & 55.69 & $\begin{array}{l}\text { Variovorax paradoxus } \\
\text { EPS }\end{array}$ & NR_074646 & 99 \\
\hline $\begin{array}{l}\text { Rhizobium legumino- } \\
\text { sarum 20-OD2 }\end{array}$ & KU647213 & 5.69 & 10.78 & Rhizobium sp. SG6 & LC042447 & 99 \\
\hline $\begin{array}{l}\text { Rhodanobacter sp. } \\
\text { 21-Y7 }\end{array}$ & KU647214 & 7.72 & 2.58 & $\begin{array}{l}\text { Rhodanobacter sp. } \\
\text { GR14-4 }\end{array}$ & FJ821729 & 99 \\
\hline $\begin{array}{l}\text { Bacillus megaterium } \\
22-\mathrm{A} 1\end{array}$ & KU647215 & 5.00 & 100.51 & Bacillus sp. B2(2010b) & HM104462 & 99 \\
\hline $\begin{array}{l}\text { Pseudomonas frederiks- } \\
\text { bergensis 23-D2 }\end{array}$ & KU647216 & 5.20 & 63.41 & Pseudomonas sp. WS06 & JN210901 & 99 \\
\hline $\begin{array}{l}\text { Bacillus megaterium } \\
24-Y 916\end{array}$ & KU647217 & 4.79 & 109.39 & Bacillus sp. BDH23 & KF933618 & 99 \\
\hline $\begin{array}{l}\text { Rhodanobacter sp. } \\
\text { 25-Y8 }\end{array}$ & KU647218 & 4.82 & 18.20 & $\begin{array}{l}\text { Rhodanobacter sp. } \\
\text { GR14-4 }\end{array}$ & FJ821729 & 99 \\
\hline $\begin{array}{l}\text { Bacillus megaterium } \\
26-Y 91\end{array}$ & KU647219 & 4.63 & 46.61 & $\begin{array}{l}\text { Bacillus megaterium } \\
\text { HNS79 }\end{array}$ & KF933676 & 99 \\
\hline $\begin{array}{l}\text { Bacillus megaterium } \\
27-Y 93\end{array}$ & KU647220 & 4.37 & 117.30 & Bacillus sp. NyZ44 & HQ231223 & 99 \\
\hline $\begin{array}{l}\text { Bacillus megaterium } \\
\text { 28-Y911 }\end{array}$ & KU647221 & 4.54 & 126.48 & $\begin{array}{l}\text { Bacillus megaterium } \\
\text { Bacteria I }\end{array}$ & KT427436 & 99 \\
\hline
\end{tabular}


Table 2 continued

\begin{tabular}{|c|c|c|c|c|c|c|}
\hline \multirow[t]{2}{*}{ Strain } & \multirow[t]{2}{*}{ Accession number } & \multirow[t]{2}{*}{$\mathrm{pH}$ of medium } & \multirow{2}{*}{$\begin{array}{l}\text { Soluble-P concentra- } \\
\text { tion }\left(\mu \mathrm{g} \mathrm{mL}^{-1}\right)\end{array}$} & \multicolumn{3}{|l|}{ Closest reference strain } \\
\hline & & & & Affiliation & Accession number & Similarity (\%) \\
\hline $\begin{array}{l}\text { Bacillus megaterium } \\
29-Y 924\end{array}$ & KU647222 & 4.55 & 136.83 & Bacillus sp. BDH4 & KF933626 & 99 \\
\hline $\begin{array}{l}\text { Bacillus megaterium } \\
\text { 30-Y1411 }\end{array}$ & KU647223 & 4.48 & 134.39 & Bacillus sp. WXGRY7 & KJ184905 & 99 \\
\hline $\begin{array}{l}\text { Bacillus megaterium } \\
31-Y 142\end{array}$ & KU647224 & 4.71 & 97.29 & $\begin{array}{l}\text { Bacillus megaterium } \\
\text { Bacteria I }\end{array}$ & KT427436 & 99 \\
\hline $\begin{array}{l}\text { Arthrobacter sp. } \\
\text { 32-OD9 }\end{array}$ & KU647225 & 5.31 & 43.19 & Arthrobacter sp. WS20 & JN899573 & 99 \\
\hline $\begin{array}{l}\text { Streptomyces tumes- } \\
\text { cens } 33-\mathrm{X} 1\end{array}$ & KU647226 & 8.02 & 2.77 & $\begin{array}{l}\text { Streptomyces tumes- } \\
\text { cens OTP-4-2 }\end{array}$ & AF346485 & 99 \\
\hline $\begin{array}{l}\text { Streptomyces prasinopi- } \\
\text { losus } 34-Y 1\end{array}$ & KU647227 & 7.75 & 3.07 & Streptomyces sp. GS15 & JX679244 & 99 \\
\hline $\begin{array}{l}\text { Streptomyces rishiriensis } \\
35-Y 3\end{array}$ & KU647228 & 5.76 & 44.37 & $\begin{array}{l}\text { Streptomyces sp. } \\
\text { YRA147 }\end{array}$ & JX430828 & 99 \\
\hline Kurthia zopfii 36-Y7 & KU647229 & 4.52 & 81.57 & $\begin{array}{l}\text { Bacillus thermophilus } \\
\text { SgZ-10 }\end{array}$ & NR_109677 & 97 \\
\hline $\begin{array}{l}\text { Rhodanobacter sp. } \\
\text { 37-Y8 }\end{array}$ & KU647230 & 4.88 & 32.55 & $\begin{array}{l}\text { Rhodanobacter sp. } \\
\text { GR14-4 }\end{array}$ & FJ821729 & 99 \\
\hline $\begin{array}{l}\text { Bacillus megaterium } \\
\text { 38-Y92 }\end{array}$ & KU647231 & 4.51 & 91.04 & $\begin{array}{l}\text { Bacillus megaterium } \\
\text { HNS88 }\end{array}$ & KF933685 & 99 \\
\hline $\begin{array}{l}\text { Bacillus megaterium } \\
\text { 39-Y94 }\end{array}$ & KU647232 & 4.43 & 91.62 & $\begin{array}{l}\text { Bacillus megaterium } \\
\text { HNS79 }\end{array}$ & KF933676 & 99 \\
\hline $\begin{array}{l}\text { Bacillus megaterium } \\
\text { 40-Y95 }\end{array}$ & KU647233 & 4.44 & 134.49 & Bacillus sp. S10 & HE662645 & 99 \\
\hline $\begin{array}{l}\text { Bacillus megaterium } \\
41-Y 99\end{array}$ & KU647234 & 4.41 & 159.48 & $\begin{array}{l}\text { Bacillus megaterium } \\
\text { BS17 }\end{array}$ & KR063197 & 99 \\
\hline $\begin{array}{l}\text { Bacillus megaterium } \\
42-Y 910\end{array}$ & KU647235 & 4.58 & 75.22 & Bacillus megaterium B2 & KT307979 & 99 \\
\hline $\begin{array}{l}\text { Bacillus megaterium } \\
43-Y 912\end{array}$ & KU647236 & 4.58 & 72.39 & Bacillus sp. BDH23 & KF933618 & 99 \\
\hline $\begin{array}{l}\text { Bacillus megaterium } \\
44-Y 913\end{array}$ & KU647237 & 4.50 & 46.51 & $\begin{array}{l}\text { Bacillus megaterium } \\
\text { HNS88 }\end{array}$ & KF933685 & 99 \\
\hline $\begin{array}{l}\text { Bacillus megaterium } \\
\text { 45-Y914 }\end{array}$ & KU647238 & 4.65 & 94.26 & $\begin{array}{l}\text { Bacillus megaterium } \\
\text { ML482 }\end{array}$ & KC692173 & 99 \\
\hline $\begin{array}{l}\text { Bacillus megaterium } \\
46-Y 923\end{array}$ & KU647239 & 4.62 & 81.57 & Bacillus sp. BDH4 & KF933626 & 99 \\
\hline $\begin{array}{l}\text { Bacillus megaterium } \\
47-Y 141\end{array}$ & KU647240 & 4.62 & 70.73 & Bacillus sp. BSp-2 & KF835394 & 99 \\
\hline Rhizobium sp. 48-Y930 & KU647241 & 7.86 & 3.75 & Rhizobium sp. CC-SKC2 & HQ113369 & 99 \\
\hline $\begin{array}{l}\text { Bacillus megaterium } \\
49-Y 1412\end{array}$ & KU647242 & 4.60 & 138.68 & $\begin{array}{l}\text { Bacillus megaterium } \\
\text { HNS88 }\end{array}$ & KF933685 & 99 \\
\hline $\begin{array}{l}\text { Rhizobium sp. } \\
50-Y 1414\end{array}$ & KU647243 & 8.02 & 5.60 & Rhizobium sp. CC-SKC2 & HQ113369 & 99 \\
\hline $\begin{array}{l}\text { Burkholderia cepacia } \\
51-Y 1415\end{array}$ & KU647244 & 5.00 & 2.03 & Burkholderia sp. xin-1 & KF059269 & 99 \\
\hline $\begin{array}{l}\text { Arthrobacter defluvii } \\
\text { 52-OD12 }\end{array}$ & KU647245 & 4.59 & 76.10 & $\begin{array}{l}\text { Uncultured bacterium } \\
\text { D1-57 }\end{array}$ & KC554872 & 99 \\
\hline $\begin{array}{l}\text { Bacillus acidiceler } \\
\text { 53-Q11 }\end{array}$ & KU647246 & 4.39 & 127.07 & Bacillus sp. S21001 & D84560 & 99 \\
\hline $\begin{array}{l}\text { Streptomyces prasinopi- } \\
\text { losus } 54-Y 1\end{array}$ & KU647247 & 5.29 & 49.64 & Streptomyces sp. GS15 & JX679244 & 99 \\
\hline $\begin{array}{l}\text { Pseudomonas frederiks- } \\
\text { bergensis 55-D3 }\end{array}$ & KU647248 & 4.96 & 35.87 & $\begin{array}{l}\text { Pseudomonas sp. } \\
\text { B3039 }\end{array}$ & KC236870 & 99 \\
\hline $\begin{array}{l}\text { Burkholderia phytofir- } \\
\text { mans 56-OY3 }\end{array}$ & KU647249 & 8.20 & 3.85 & Burkholderia sp. C2-14 & JF900054 & 99 \\
\hline
\end{tabular}


Table 2 continued

\begin{tabular}{|c|c|c|c|c|c|c|}
\hline \multirow[t]{2}{*}{ Strain } & \multirow[t]{2}{*}{ Accession number } & \multirow[t]{2}{*}{$\mathrm{pH}$ of medium } & \multirow{2}{*}{$\begin{array}{l}\text { Soluble-P concentra- } \\
\text { tion }\left(\mu \mathrm{g} \mathrm{mL}^{-1}\right)\end{array}$} & \multicolumn{3}{|l|}{ Closest reference strain } \\
\hline & & & & Affiliation & Accession number & Similarity (\%) \\
\hline $\begin{array}{l}\text { Variovorax paradoxus } \\
57-Y 925\end{array}$ & KU647250 & 5.30 & 10.88 & Variovorax sp. LZA10 & GQ861460 & 99 \\
\hline Telluria mixta 58-Y97 & KU647251 & 4.62 & 106.85 & $\begin{array}{l}\text { Uncultured bacterium } \\
\text { SuR5 }\end{array}$ & AB608684 & 99 \\
\hline $\begin{array}{l}\text { Sphingomonas koreen- } \\
\text { sis 59-Y96 }\end{array}$ & KU647252 & 7.31 & 2.77 & $\begin{array}{l}\text { Uncultured Sphingo- } \\
\text { monas sp. Plot4-G09 }\end{array}$ & EU449628 & 99 \\
\hline $\begin{array}{l}\text { Streptomyces flaveolus } \\
60-0 D 3\end{array}$ & KU647253 & 7.95 & 2.19 & $\begin{array}{l}\text { Streptomyces flaveolus } \\
\text { NRRL B-1334 }\end{array}$ & NR_116094 & 99 \\
\hline $\begin{array}{l}\text { Rhodanobacter sp. } \\
\text { 61-Y8 }\end{array}$ & KU647254 & 4.49 & 62.23 & $\begin{array}{l}\text { Rhodanobacter sp. } \\
\text { GR14-4 }\end{array}$ & FJ821729 & 99 \\
\hline $\begin{array}{l}\text { Streptomyces sp. } \\
62-Y 930\end{array}$ & KU647255 & 6.50 & 3.46 & $\begin{array}{l}\text { Streptomyces sp. } \\
\text { N4-145 }\end{array}$ & EF063495 & 99 \\
\hline $\begin{array}{l}\text { Rhodococcus cer- } \\
\text { cidiphylli 63-OD5 }\end{array}$ & KU647256 & 6.77 & 3.07 & Rhodococcus sp. AB73 & KC019201 & 98 \\
\hline $\begin{array}{l}\text { Bacillus megaterium } \\
64-\mathrm{Y} 98\end{array}$ & KU647257 & 4.53 & 107.44 & Bacillus sp. BDH23 & KF933618 & 100 \\
\hline $\begin{array}{l}\text { Bacillus megaterium } \\
65-\text { Y918 }\end{array}$ & KU647258 & 4.71 & 69.75 & Bacillus megaterium B2 & KT307979 & 99 \\
\hline $\begin{array}{l}\text { Bacillus megaterium } \\
66-Y 143\end{array}$ & KU647259 & 4.55 & 82.84 & $\begin{array}{l}\text { Bacillus megaterium } \\
\text { Bacteria I }\end{array}$ & KT427436 & 99 \\
\hline $\begin{array}{l}\text { Rhodococcus sp. } \\
\text { 67-OD10 }\end{array}$ & KU647260 & 5.45 & 52.67 & $\begin{array}{l}\text { Uncultured bacterium } \\
\text { clone Md-133 }\end{array}$ & KT905708 & 99 \\
\hline $\begin{array}{l}\text { Arthrobacter oxydans } \\
68 \text {-OY1 }\end{array}$ & KU647261 & 6.15 & 16.44 & $\begin{array}{l}\text { Arthrobacter oxydans } \\
\text { BGSLP35 }\end{array}$ & KP192013 & 99 \\
\hline $\begin{array}{l}\text { Pseudomonas sp. } \\
\text { 69-Y94 }\end{array}$ & KU647262 & 4.87 & 71.51 & $\begin{array}{l}\text { Pseudomonas sp. } \\
\text { B3042 }\end{array}$ & KC236872 & 99 \\
\hline $\begin{array}{l}\text { Bacillus megaterium } \\
70-Y 917\end{array}$ & KU647263 & 4.43 & 76.10 & Bacillus sp. S10 & HE662645 & 99 \\
\hline $\begin{array}{l}\text { Pseudomonas sp. } \\
\text { 71-Y928 }\end{array}$ & KU647264 & 5.41 & 37.82 & $\begin{array}{l}\text { Pseudomonas sp. } \\
\text { B3042 }\end{array}$ & KC236872 & 99 \\
\hline $\begin{array}{l}\text { Bacillus megaterium } \\
72-Y 13\end{array}$ & KU647265 & 4.61 & 112.03 & $\begin{array}{l}\text { Bacillus megaterium } \\
\text { HNS79 }\end{array}$ & KF933676 & 99 \\
\hline $\begin{array}{l}\text { Bacillus megaterium } \\
\text { 73-Y142 }\end{array}$ & KU647266 & 4.77 & 106.46 & $\begin{array}{l}\text { Bacillus megaterium } \\
\text { HNS88 }\end{array}$ & KF933685 & 99 \\
\hline $\begin{array}{l}\text { Streptomyces sp. } \\
74-Y 144\end{array}$ & KU647267 & 5.00 & 22.50 & $\begin{array}{l}\text { Streptomyces sp. } \\
\text { N4-145 }\end{array}$ & EF063495 & 99 \\
\hline $\begin{array}{l}\text { Leifsonia shinshuensis } \\
\text { 75-Y145 }\end{array}$ & KU647268 & 4.54 & 27.08 & $\begin{array}{l}\text { Leifsonia shinshuensis } \\
\text { DB } 102\end{array}$ & NR_043663 & 99 \\
\hline $\begin{array}{l}\text { Bacillus megaterium } \\
76-Y 149\end{array}$ & KU647269 & 4.78 & 59.70 & Bacillus sp. BSp-2 & KF835394 & 99 \\
\hline $\begin{array}{l}\text { Streptomyces sp. } \\
77-Y 1410\end{array}$ & KU647270 & 5.25 & 34.41 & $\begin{array}{l}\text { Streptomyces sp. } \\
\quad \text { N4-145 }\end{array}$ & EF063495 & 99 \\
\hline
\end{tabular}

above. The P solubilizing percentage (\%) was calculated as follows,

$$
\text { P solubilizing percentage }=\frac{P_{\text {free }}}{P_{\text {total }}}
$$

$\mathrm{P}_{\text {free }}$ indicated the free solubilizing phosphate concentration $\left(\mu \mathrm{g} \mathrm{mL} \mathrm{m}^{-1}\right)$ in the supernatant of liquid medium, $\mathrm{P}_{\text {total }}$ indicated the total $\mathrm{P}$ concentration $\left(\mu \mathrm{g} \mathrm{mL}^{-1}\right)$ in the liquid medium. The amounts of the organic acids, including lactic, acetic, propionic, gluconic, succinic, oxalic and citric acids, were determined using ion chromatography (ICS-3000, Dionex, USA) as previously described ( $\mathrm{Hu}$ et al. 2009). The reference standards of the corresponding sodium salts of these organic acids were chromatographically pure (Sigma-Aldrich, Shanghai, China).

\section{Statistical analyses}

Figures were generated using Microsoft Office 365. The sequences were aligned and the phylogenetic trees were constructed and annotated using Clustal X 2.0 (Larkin 
et al. 2007), MEGA 6.0 (Tamura et al. 2013) and iTOL v3 (Letunic and Bork 2016), respectively. The correlations and variance analyses (ANOVAs) used IBM SPSS Statistics 21 .

\section{Results}

\section{Rapid screening of the iPSB strains}

Six 96-well microplates (576 wells in total) with modified PVK were used for screening the two soil samples for iPSBs (Fig. 2a). We isolated 39 and 35 iPSB strains from the Hailun and Yingtan soils, respectively, and two uncertain bacterial strains were obtained from the Hailun soil. The uncertain strains were transferred to 200mm PVK medium plates (Fig. 2b), where they survived and produced slight color changes and clearance zones. We thus classified these two strains as iPSB strains. The other iPSB strains produced obvious color changes after inoculation (Fig. 2c). Hence, a total of 76 iPSB strains were obtained with an average screening efficiency of $13.19 \pm 1.47 \%$. Only one bacterial strain survived in each microplate well, which was confirmed by streaking onto 200-mm PVK medium plates. Fungal contamination was well confined to single wells (Fig. 2a, purple circle).

\section{Phylogenetic identification of iPSB strains}

The maximum-likelihood phylogenetic trees based on $16 \mathrm{~S}$ rRNA sequences from the $76 \mathrm{iPSB}$ strains are

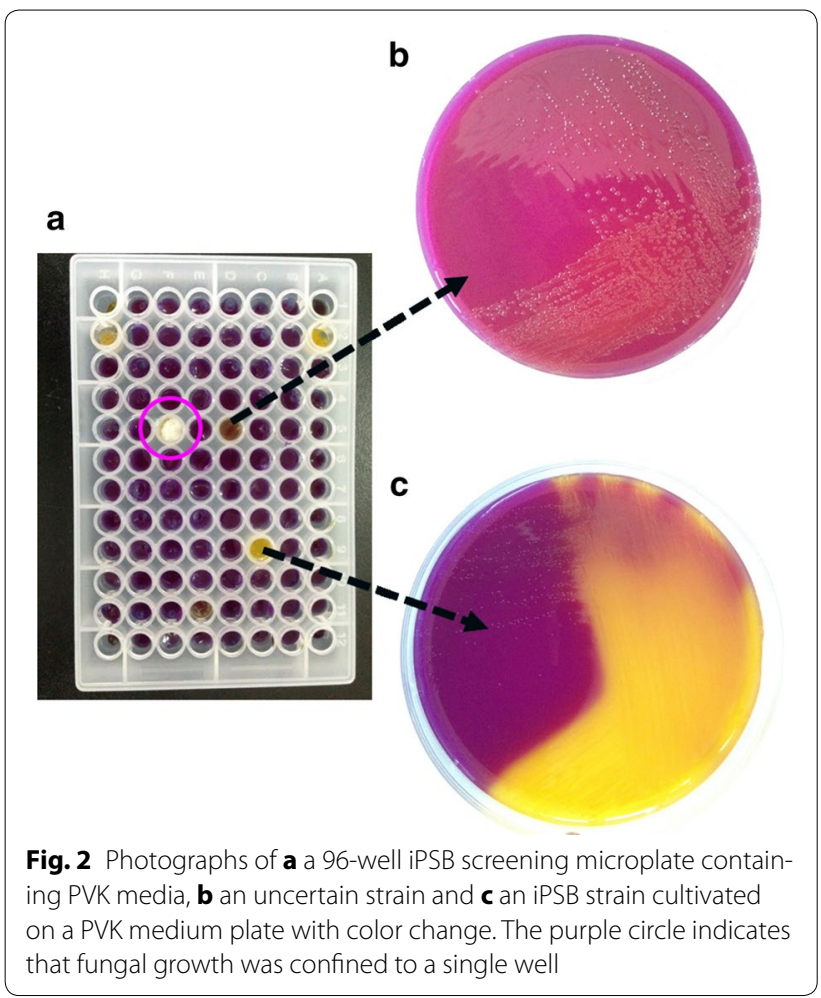

presented in Fig. 3. Five phyla were identified: Actinobacteria, Firmicutes and $\alpha$-, $\beta$ - and $\gamma$-Proteobacteria. Bacillus megaterium was the most dominant species (32 isolates). The other 44 isolates were eight Arthrobacter sp., seven Streptomyces sp., seven Pseudomonas sp., four Rhodanobacter sp., three Rhizobium sp., three Rhodococcus sp., two Burkholderia sp., two Variovorax paradoxus and single isolate of B. acidceler, Duganella sp., Kurthia zopfi, Leifsonia shinshuensis, Massilia sp., Pseudoduganella sp., Sphingomonas koreensis and Tellura mixta. The closest phylogenic reference strains with their similarities are listed in Table 2. Interestingly, K. zopfii 36-Y7 was only 97\% similar to the closest strain based on the reference sequence from the GenBank database.

\section{Biochemical characterization of the iPSB strains}

Details of the medium $\mathrm{pH}$ and soluble-P concentration after $72 \mathrm{~h}$ incubation are shown in Fig. 3 and Table 2. The $\mathrm{pH}$ decreased as the soluble- $\mathrm{P}$ concentration increased in the liquid medium. The $\mathrm{pH}$ for $B$. megaterium 27-Y93 decreased from an initial 7.0 to 4.37 after $72 \mathrm{~h}$. Interestingly, the $\mathrm{pH}$ for Arthrobacter defluvii 06-OD12, Streptomyces tumescens 33-X1, Rhizobium sp. 48-Y930, Rhizobium sp. 50-Y1414, Burkholderia phytofirmans 56-OY3 and Streptomyces flaveolus 60-OD3 increased to $8.34,8.02,7.86,8.02,8.20$ and 7.95 , respectively. The soluble-P concentration ranged from 2.03 to $159.48 \mu \mathrm{g} \mathrm{mL}^{-1}$. Neither soluble P nor a decrease in $\mathrm{pH}$ was detected in the control treatment. The soluble-P concentration was highest for $B$. megaterium Y99 $\left(159.48 \mu \mathrm{g} \mathrm{mL}{ }^{-1}\right)$, followed by $B$. megaterium Y1412 (138.68 $\left.\mu \mathrm{g} \mathrm{mL} \mathrm{m}^{-1}\right), B$. megaterium $\mathrm{Y} 924\left(136.83 \mu \mathrm{g} \mathrm{mL} \mathrm{L}^{-1}\right)$ and $B$. megaterium Y95 $\left(134.49 \mu \mathrm{g} \mathrm{mL}^{-1}\right)$, and their medium $\mathrm{pH}$ decreased to about 4.5. These four strains were further analyzed as representative iPSB strains (Fig. 4a-d). Medium pH was significantly negatively correlated with soluble-P concentration $(P<000.1)$ (Fig. 5a).

\section{Growth analysis of the four representative iPSB strains}

The four representative iPSB strains were incubated with PVK at $30^{\circ} \mathrm{C}$ for $168 \mathrm{~h}$. The organic acids were identified by ion chromatography (Table 3). Only three of the seven organic acids (succinic, oxalic and citric) were detected. Among these, the concentration of succinic acid peaked at $209 \mathrm{ppm}$, which was significantly higher than the concentrations of the other organic acids $(P<0.05)$.

We further analyzed the relationship between the soluble-P and succinic acid concentrations for these four strains (Fig. 4). Both concentrations increased with bacterial growth. A linear regression analysis indicated that the amount of soluble $P$ was significantly positively correlated with the release of succinic acid $(P<0.001)$ (Fig. 5b). 

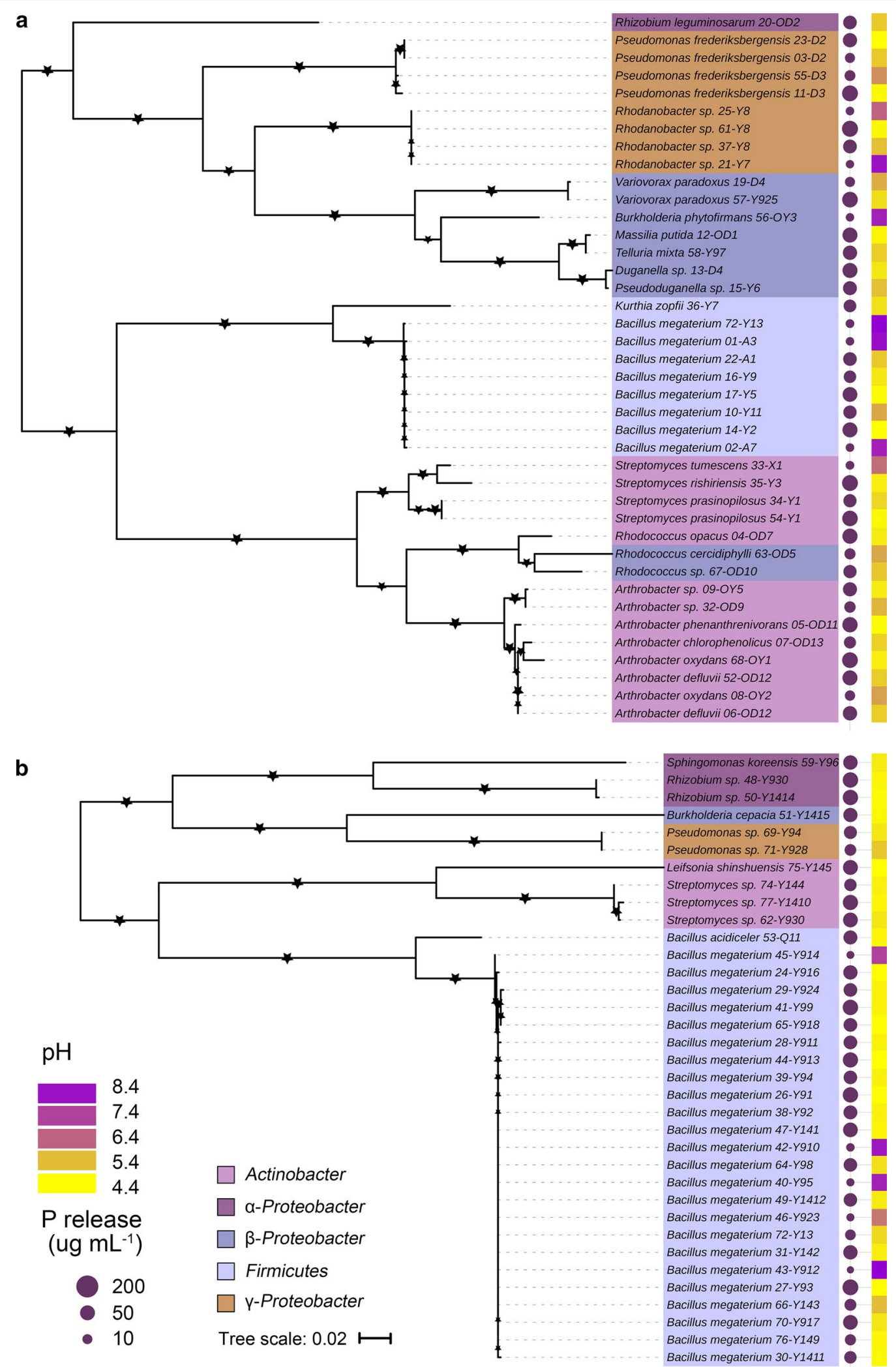

Fig. 3 Phylogenic trees of 76 iPSB strains from $\mathbf{a}$ Hailun and $\mathbf{b}$ Yingtan soil based on their $16 \mathrm{~S}$ rRNA sequences. Maximum likelihood was used to construct the trees with bootstrapping (1000 replicates). Bootstrap percentages $>50 \%$ are marked with stars. The scale bar indicates 0.02 accumulated changes per nucleotide position 

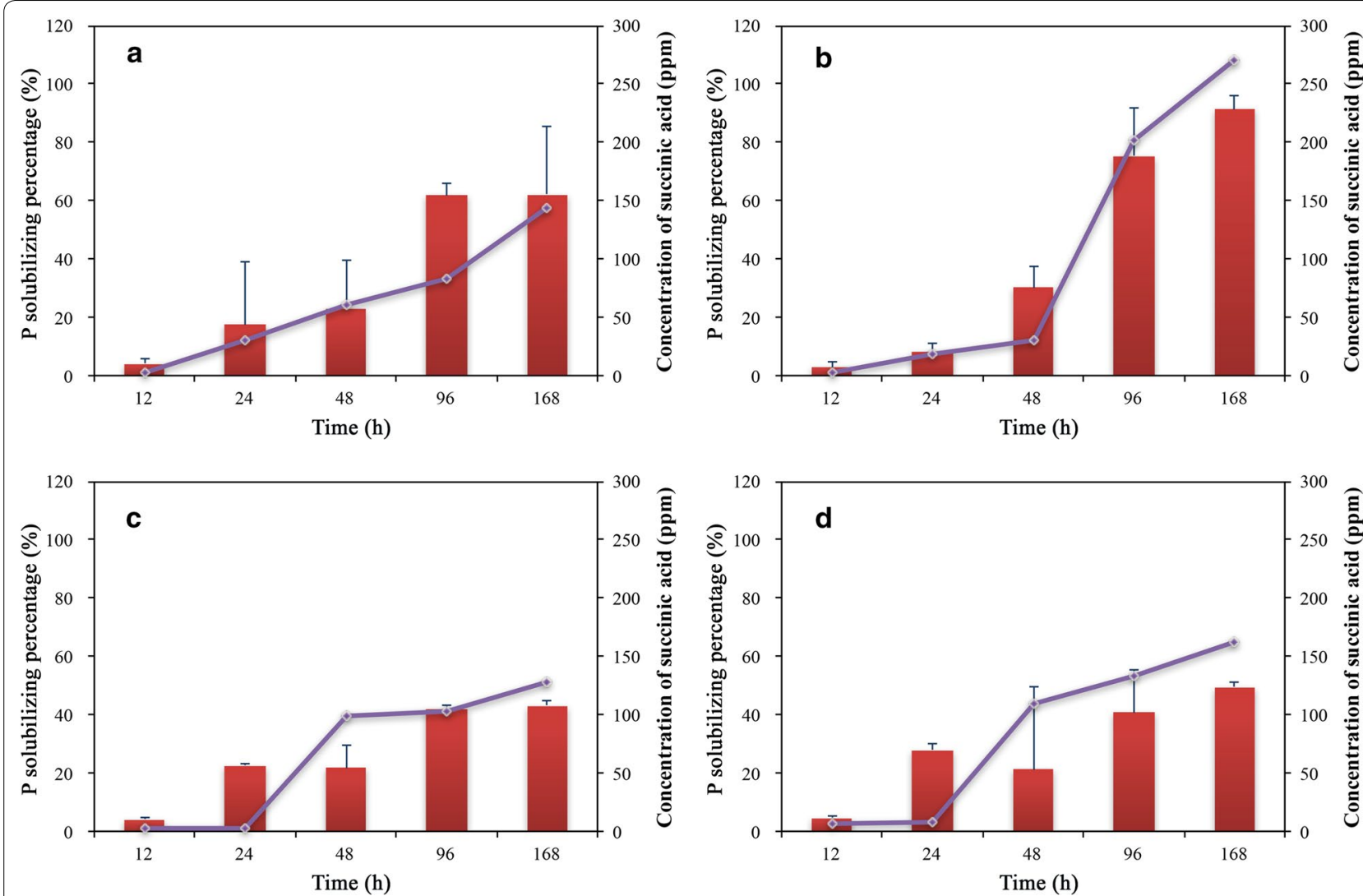

Fig. 4 The P-solubilizing percentages (red bars) and succinic acid production (purple lines) for the four representative iPSB strains over $168 \mathrm{~h}$ of growth. a B. megaterium $Y 1412$, b B. megaterium Y99, c B. megaterium Y95 and d B. megaterium Y924. Each value represents the mean of three replicates \pm standard deviation

\section{Discussion}

Current agricultural practices tend to use sustainable technology, including the use of biofertilizers with multiple functions, to achieve high crop yields, which requires high soluble-P concentrations. iPSBs thus play an essential role in releasing $\mathrm{P}$ from soil-fixation systems and in preserving enough free phosphate in the rhizosphere for plant uptake and growth. iPSB inoculation can promote plant growth (Kaur and Reddy 2015; Yu et al. 2012), so identifying highly efficient iPSB strains thus becomes important. Our rapid screening using 96-well microplates provided an efficient method for simultaneously isolating numerous iPSB strains. The method isolated 76 positive iPSB strains within $72 \mathrm{~h}$ from two soils with three replicates. Each well has limited space, so only a single bacterial strain may survive if the sample is sufficiently diluted. Each well in our study contained a sole iPSB strain with a $10^{5}$ dilution of soil sample from two remote sites with different chemical properties, which was verified by incubation on $200-\mathrm{mm}$ medium plates (Fig. 2), suggesting that further purification by a second or third colony transfer may not be needed. Positive iPSB strains were also easy to observe and count using bromocresol purple as an indicator.

Wells without bacterial growth and a color change were deemed to be negative, and yellow wells (definitely with bacterial growth) were deemed to be positive. Only the uncertain or ambiguous wells may need further confirmation. Two slightly yellow wells in our study were ultimately demonstrated to be positive iPSB strains with P-solubilizing ability. Our method can also effectively prevent the spread of fungal contamination. Most screening media contain glucose or other carbohydrates as carbon sources, so heterologous fungal spores can easily grow or be initially introduced in the diluted sample. Once a single fungal colony appears, it can promptly spread throughout the medium plate and interfere with screening. The first screening of iPSBs from soil samples also may need more than $72 \mathrm{~h}$ of incubation, so fungal survival is likely. Fungal spores in our method (Fig. 2a, purple circle), however, were confined to only one well instead of spreading and affecting surrounding wells. The rapid screening with 96-well microplates was demonstrated to be an efficient and effective way to isolate iPSB strains. 

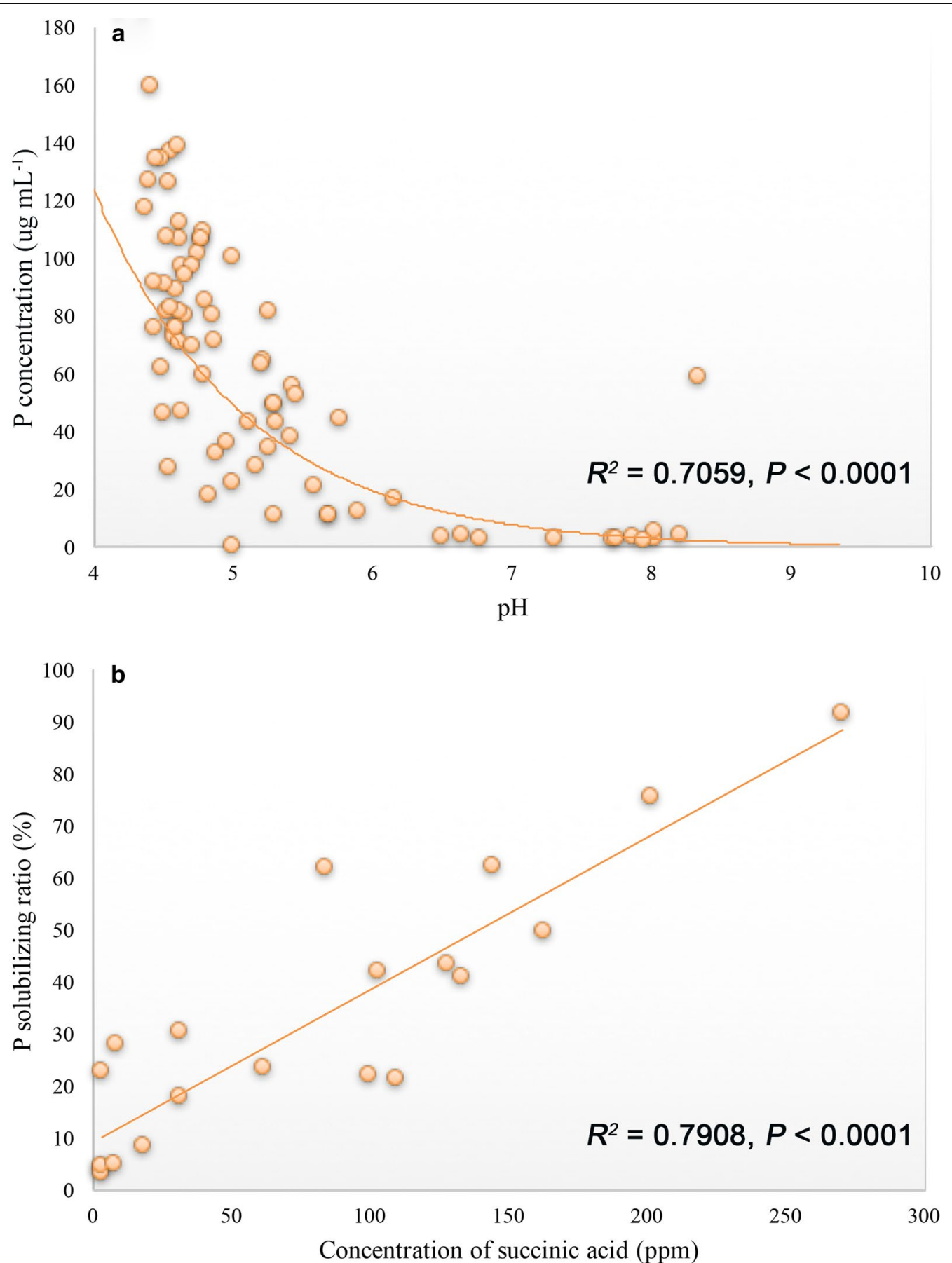

Fig. 5 Regression analysis of $\mathbf{a}$ the correlation between $\mathrm{pH}$ and P release for all iPSB strains (regression function: $y=4926.1 \mathrm{e}^{-0.922 x}$ ) and $\mathbf{b}$ the correlation between the concentration of succinic acid and the P-solubilizing percentage for the four representative iPSB strains (regression function: $y=0.2925 x+9.3694)$

The identification of iPSB strains based on 16S rRNA sequences illustrated the phylogenetic structure of the potential iPSB communities in the two soils. B. megaterium contributed $>30 \%$ to the total iPSB population, which was also most frequently found in the Yingtan soil with an acidic pH (Fig. 3). Bacillus is abundant in various types of soil and has a strong P-solubilizing ability in cropland (Chen et al. 2006; Karagöz et al. 2012; Oliveira et al. 2009; Xuan et al. 2011). The next two most common genera, Arthrobacter and Streptomyces, which 
Table 3 Amounts of organic acids secreted by the four reference iPSB strains after incubation for $168 \mathrm{~h}$

\begin{tabular}{|c|c|c|c|c|c|c|c|}
\hline \multirow[t]{2}{*}{ iPSB strain } & \multicolumn{7}{|c|}{ Organic acid (ppm) } \\
\hline & Lactic & Acetic & Propionic & Gluconic & Succinic & Oxalic & Citric \\
\hline B. megaterium Y95 & ND & ND & ND & ND & $202.30 \pm 105.27$ & $6.66 \pm 1.22$ & $2.33 \pm 0.89$ \\
\hline B. megaterium Y99 & ND & ND & ND & ND & $196.60 \pm 99.77$ & $6.53 \pm 0.06$ & $0.24 \pm 0.02$ \\
\hline B. megaterium Y924 & ND & ND & ND & ND & $208.88 \pm 91.90$ & $5.21 \pm 2.10$ & $4.12 \pm 0.51$ \\
\hline B. megaterium $Y 1412$ & ND & ND & ND & ND & $142.21 \pm 27.42$ & $6.23 \pm 0.83$ & $2.37 \pm 1.67$ \\
\hline
\end{tabular}

ND not detected

belong to Actinobacteria, are common in alkaline soils where considerable $\mathrm{P}$ is released (Aislabie et al. 2006; Gopalakrishnan et al. 2011; Xiong et al. 2012). Pseudomonas is a known iPSB genus and has been well studied due to genetic evidence of inorganic-P solubilization (Babu-Khan et al. 1995; Kwak et al. 2015; Umezawa et al. 2015). Other strains rarely act as iPSBs but can promote plant growth in various types of soils (Richardson et al. 2009; Rodriguez and Fraga 1999; Zhao et al. 2014).

In our study, B. megaterium was observed to solubilize more $\mathrm{P}$ than the other genera, including Streptomyces, Arthrobacter and Pseudomonas. The presence of $B$. megaterium in the rhizosphere could thus benefit plant $\mathrm{P}$ assimilation. B. megaterium isolates Y95, Y99, Y924 and Y1412 release $>130 \mu \mathrm{g} \mathrm{mL}^{-1}$ soluble $\mathrm{P}$ when cultured in liquid PVK. Lactic and propionic acids were reported to be the two most common organic acids secreted by $B$. megaterium in a previous study (Chen et al. 2006), but we did not observe any ion-chromatographic peaks in the corresponding retention times of these two organic acids (Table 3). Gluconic acid has also been considered an important organic acid for P release (Rodríguez et al. 2006), but we did not detect this acid for any of the four representative iPSB strains. Of the three secreted organic acids, citric and oxalic acids are efficient P-solubilizing acids (Bolan et al. 1994), but their amounts released by these four strains were relatively low and may not be sufficient to liberate much $\mathrm{P}$. The dominant succinic acid was not a common organic acid for $\mathrm{P}$ release, but the amounts secreted were strongly correlated with P release for the four representative strains over time (Fig. 4). Succinic acid concentration was strongly, positively and linearly correlated with $\mathrm{P}$ release (Fig. 5a) $\left(R^{2}=0.7908\right.$, $P<0.001)$, suggesting that succinic acid was the main solubilizing acid secreted by $B$. megaterium.

Environmental $\mathrm{pH}$ and organic acid secretion were two common factors accounting for the mobilization of available P. pH is usually negatively correlated with the release of P (Chen et al. 2006; Rodriguez and Fraga 1999). The $\mathrm{pH}$ of the medium for some of the iPSBs in our study, however, was alkaline (Table 2), and the iPSBs likely had the ability to release $P$ because they survived well at a high cell density (data not shown). The regression analysis of medium $\mathrm{pH}$ and $\mathrm{P}$ release (Fig. 5a) indicated a negative but not linear correlation. The $\mathrm{pH}$ may only sharply decrease with an increase in $\mathrm{P}$ release at acidic pHs, suggesting that soil $\mathrm{pH}$ may not be a universal factor to account for microbial P solubilization by iPSB stains.

We demonstrated the efficiency and effectiveness of this rapid iPSB screening method using 96-well microplates. This method is rapid and easy to manipulate and observe and can prevent the spread of fungal growth. $B$. megaterium was the main iPSB strain but released mostly succinic acid rather than other common organic acids for P solubilization. Our study may be useful for mechanistic study of microbial inorganic-P solubilization.

\section{Abbreviations}

P: phosphorus; iPSBs: inorganic phosphate solubilizing bacteria; Olsen P: available P; PVK: Pikovskaya medium; NCBI: National Center for Biotechnology Information; ANOVA: correlations and variance analyses.

\section{Authors' contributions}

Y-GZ and X-RY conceived the project. D-PZ and Q-FB conducted the soil samplings. $\mathrm{B}-\mathrm{XZ}$ and $\mathrm{MI}$ conceived the rapid screening method. $\mathrm{H}-\mathrm{ZL}$ and G-WZ conducted the bacterial isolation and identification. B-XZ, MI and KD conducted the experiment. B-XZ, Y-GZ and JP analyzed the results and wrote the manuscript. All authors read and approved the final manuscript.

\section{Author details}

${ }^{1}$ Key Laboratory of Urban Environment and Health, Institute of Urban Environment, Chinese Academy of Sciences, Xiamen 361021, People's Republic of China. ${ }^{2}$ University of Chinese Academy of Sciences, Beijing 100049, People's Republic of China. ${ }^{3}$ Consejo Superior de Investigaciones Científicas (CSIC), Global Ecology Unit, Centre for Ecological Research and Forestry Applications (CREAF), Universitat Autònoma de Barcelona (UAB), Bellaterra, 08193 BarceIona, Catalonia, Spain. ${ }^{4}$ CREAF, Cerdanyola del Vallès, 08193 Barcelona, Catalonia, Spain. ${ }^{5}$ State Key Laboratory of Biocontrol, Key Laboratory of Biodiversity Dynamics and Conservation of Guangdong Higher Education Institutes, College of Ecology and Evolution, Sun Yat-sen University, Guangzhou 510275, People's Republic of China. ${ }^{6}$ MOE Key Laboratory of Environment Remediation and Ecological Health, College of Environmental and Resource Sciences, Zhejiang University, Hangzhou 310058, People's Republic of China. ${ }^{7}$ State Key Laboratory of Urban and Regional Ecology, Research Center for Eco-Environmental Sciences, Chinese Academy of Sciences, Beijing 100085, China.

\section{Acknowledgements}

The authors wish to acknowledge Dr. Jian-Qiang Su and Ms. Yu Yan (Institute of Urban Environment, Chinese Academy of Sciences) for their helpful suggestions and assistance with figure production.

\section{Competing interests}

The authors declare that they have no competing interests. 


\section{Availability of data and materials}

The 16S rRNA sequences were uploaded to the NCBI Sequence Read Archive (KU647195-KU647270). The biochemical properties and other analyzed data has been fully stated in this study.

\section{Consent for publication}

Not applicable.

\section{Ethics approval and consent to participate}

Not applicable.

\section{Funding}

This study was financially supported by the Strategic Priority Research Program of the Chinese Academy of Sciences (XDB15020402), the Natural Science Foundation of China $(41771285,41430858)$, the National Key Research and Development Program of China (2017YFD0200201) and the European Research Council Synergy Grant ERC-SyG-2013-610028 IMBALANCE-P.

\section{Publisher's Note}

Springer Nature remains neutral with regard to jurisdictional claims in published maps and institutional affiliations.

Received: 7 February 2018 Accepted: 17 March 2018

Published online: 27 March 2018

\section{References}

Aislabie JM, Chhour K-L, Saul DJ, Miyauchi S, Ayton J, Paetzold RF, Balks MR (2006) Dominant bacteria in soils of Marble point and Wright valley, Victoria land, Antarctica. Soil Biol Biochem 38(10):3041-3056

Babu-Khan S, Yeo TC, Martin WL, Duron MR, Rogers RD, Goldstein AH (1995) Cloning of a mineral phosphate-solubilizing gene from Pseudomonas cepacia. Appl Environ Microbiol 61(3):972-978

Bolan NS, Naidu R, Mahimairaja S, Baskaran S (1994) Influence of low-molecular-weight organic acids on the solubilization of phosphates. Biol Fertil Soils 18(4):311-319

Chen YP, Rekha PD, Arun AB, Shen FT, Lai WA, Young CC (2006) Phosphate solubilizing bacteria from subtropical soil and their tricalcium phosphate solubilizing abilities. Appl Soil Ecol 34(1):33-41

Chung H, Park M, Madhaiyan M, Seshadri S, Song J, Cho H, Sa T (2005) Isolation and characterization of phosphate solubilizing bacteria from the rhizosphere of crop plants of Korea. Soil Biol Biochem 37(10):1970-1974

Elser J, Bennett E (2011) Phosphorus cycle: a broken biogeochemical cycle. Nature 478(7367):29-31

Elser JJ, Bracken ME, Cleland EE, Gruner DS, Harpole WS, Hillebrand H, Ngai JT, Seabloom EW, Shurin JB, Smith JE (2007) Global analysis of nitrogen and phosphorus limitation of primary producers in freshwater, marine and terrestrial ecosystems. Ecol Lett 10(12):1135-1142

Farris MH, Olson JB (2007) Detection of Actinobacteria cultivated from environmental samples reveals bias in universal primers. Lett Appl Microbiol 45(4):376-381

Goldstein AH (1995) Recent progress in understanding the molecular genetics and biochemistry of calcium phosphate solubilization by gram negative bacteria. Biol Agric Hortic 12(2):185-193

Gopalakrishnan S, Humayun P, Kiran BK, Kannan IGK, Vidya MS, Deepthi K, Rupela O (2011) Evaluation of bacteria isolated from rice rhizosphere for biological control of charcoal rot of sorghum caused by Macrophomina phaseolina (Tassi) Goid. World J Microbiol Biotechnol 27(6):1313-1321

Hu JL, Lin XG, Wang JH, Chu HY, Yin R, Zhang JB (2009) Population size and specific potential of P-mineralizing and-solubilizing bacteria under long-term P-deficiency fertilization in a sandy loam soil. Pedobiologia 53(1):49-58

Jones DL, Oburger E (2011) Solubilization of phosphorus by soil microorganisms. In: Bünemann EK, Oberson A, Frossard E (eds) Phosphorus in action, 1st edn. Springer, New York

Karagöz K, Ateş F, Karagöz H, Kotan R, Çakmakçı R (2012) Characterization of plant growth-promoting traits of bacteria isolated from the rhizosphere of grapevine grown in alkaline and acidic soils. Eur J Soil Biol 50:144-150
Kaur G, Reddy MS (2015) Effects of phosphate-solubilizing bacteria, rock phosphate and chemical fertilizers on maize-wheat cropping cycle and economics. Pedosphere 25(3):428-437

Kochian LV (2012) Rooting for more phosphorus. Nature 488(7412):466-467

Kwak Y, Jung BK, Shin JH (2015) Complete genome sequence of Pseudomonas rhizosphaerae IH5T (=DSM 16299T), a phosphate-solubilizing rhizobacterium for bacterial biofertilizer. J Biotechnol 193:137-138

Larkin MA, Blackshields G, Brown NP, Chenna R, McGettigan PA, McWilliam H, Valentin F, Wallace IM, Wilm A, Lopez R, Thompson JD, Gibson TJ, Higgins DG (2007) Clustal W and Clustal X version 2.0. Bioinformatics 23(21):2947-2948

Letunic I, Bork P (2016) Interactive tree of life (iTOL) v3: an online tool for the display and annotation of phylogenetic and other trees. Nucleic Acid Res 44(W1):W242-W245

Mehta S, Nautiyal CS (2001) An efficient method for qualitative screening of phosphate-solubilizing bacteria. Curr Microbiol 43(1):51-56

Murphy J, Riley JP (1962) A modified single solution method for the determination of phosphate in natural waters. Anal Chim Acta 27:31-36

Nautiyal CS (1999) An efficient microbiological growth medium for screening phosphate solubilizing microorganisms. FEMS Microbiol Lett 170(1):265-270

Oliveira CA, Alves VMC, Marriel IE, Gomes EA, Scotti MR, Carneiro NP, Guimarães CT, Schaffert RE, Sá NMH (2009) Phosphate solubilizing microorganisms isolated from rhizosphere of maize cultivated in an oxisol of the Brazilian Cerrado Biome. Soil Biol Biochem 41(9):1782-1787

Olsen SR, Cole CV, Wantanabe FS, Dean LA (1954) Estimation of available phosphorus in soils by extraction with sodium bicarbonate. USDA, Washington

Parkinson JA, Allen SE (1975) A wet oxidation procedure suitable for the determination of nitrogen and mineral nutrients in biological material. Commun Soil Sci Plant Anal 6(1):1-11

Penuelas J, Poulter B, Sardans J, Ciais P, van der Velde M, Bopp L, Boucher O, Godderis Y, Hinsinger P, Llusia J, Nardin E, Vicca S, Obersteiner M, Janssens IA (2013) Human-induced nitrogen-phosphorus imbalances alter natural and managed ecosystems across the globe. Nat Commun 4:2934

Richardson AE, Simpson RJ (2011) Soil microorganisms mediating phosphorus availability update on microbial phosphorus. Plant Physiol 156(3):989-996

Richardson AE, Barea JM, McNeill AM, Prigent-Combaret C (2009) Acquisition of phosphorus and nitrogen in the rhizosphere and plant growth promotion by microorganisms. Plant Soil 321(1-2):305-339

Rodríguez H, Fraga R (1999) Soil microorganisms mediating phosphorus availability update on microbial phosphorus. Biotechnol Adv 17(4):319-339

Rodríguez H, Fraga R, Gonzalez T, Bashan Y (2006) Genetics of phosphate solubilization and its potential applications for improving plant growthpromoting bacteria. Plant Soil 287(1-2):15-21

Shen JP, Zhang LM, Zhu YG, Zhang JB, He JZ (2008) Abundance and composition of ammonia-oxidizing bacteria and ammonia-oxidizing archaea communities of an alkaline sandy loam. Environ Microbiol 10(6):1601-1611

Tamura K, Stecher G, Peterson D, Filipski A, Kumar S (2013) MEGA6: molecular evolutionary genetics analysis version 6.0. Mol Biol Evol 30(12):2725-2729

Umezawa K, Takeda K, Ishida T, Sunagawa N, Makabe A, Isobe K, Koba K, Ohno H, Samejima M, Nakamura N, Igarashi K, Yoshida M (2015) A novel pyrroloquinoline quinone-dependent 2-keto-D-glucose dehydrogenase from Pseudomonas aureofaciens. J Bacteriol 197(8):1322-1329

van der Heijden MG, Bardgett RD, van Straalen NM (2008) The unseen majority: soil microbes as drivers of plant diversity and productivity in terrestrial ecosystems. Ecol Lett 11(3):296-310

Xiong J, Liu Y, Lin X, Zhang H, Zeng J, Hou J, Yang Y, Yao T, Knight R, Chu H (2012) Geographic distance and $\mathrm{pH}$ drive bacterial distribution in alkaline lake sediments across Tibetan Plateau. Environ Microbiol 14(9):2457-2466

Xuan Y, Xu L, Tian HZ, Liu GH, Cui M (2011) Isolation and characterization of phosphate-solubilizing bacteria from walnut and their effect on growth and phosphorus mobilization. Biol Fertil Soils 47(4):437-446

Yu X, Liu X, Zhu TH, Liu GH, Mao C (2012) Co-inoculation with phosphate-solubilzing and nitrogen-fixing bacteria on solubilization of rock phosphate and their effect on growth promotion and nutrient uptake by walnut. Eur J Soil Biol 50:112-117

Zhao K, Penttinen P, Zhang XP, Ao XL, Liu MK, Yu XM, Chen Q (2014) Maize rhizosphere in Sichuan, China, hosts plant growth promoting Burkholderia cepacia with phosphate solubilizing and antifungal abilities. Microbiol Res 169(1):76-82 